Arch. Tierz., Dummerstorf 43 (2000) 6, 621-631 Aus der Universitat Rostock, Institut fur umweltgerechte Tierhaltung', Rostock und dem Forschungsinstitut fur die
Biologie Inndwirtschaflicher Nutztiere (FBN), Dummerstorf ${ }^{2}$

HOLGER KLUTH', MARTIN GABEL', JÜRGEN VOIGT ${ }^{2}$ und ULRIKE SCHÖNHUSEN ${ }^{2}$

\title{
Verwertung der im Intestinum wachsender Bullen absorbierten essentiellen Aminosäuren
}

\author{
Herrn Professor Dr. habil. agr. H. Hagemeister zum 65. Geburtstag gewidmet
}

\begin{abstract}
Summary Title of the paper: The efficiency of utilization of intestinal digestible indispensable amino acids in growing
bulls

There are problems to determine the efficiency of protein deposition of intestinal absorbed amino acids, because it cannot be excluded that the amount of single or some indispensable amino acid (IAA) can influence the growing performance of the animal. The dependence of amino acid gain on the flux of essential absorbable amino acids into the duodenum was studied in growing bulls to calculate the utilization rate of these amino acids. Four growing bulls (Schwarzbuntes Milchrind, BW: $240-310 \mathrm{~kg}$ ) with duodenal T-type cannulas were fed diets varying in crude protein content. Energy level was $11,2 \mathrm{MJ} \mathrm{ME} / \mathrm{kg} \mathrm{DM}$. The efficiency for the different absorbable IAA was decreased with increasing nutritional level. This is in contrast with the constant utilization factor of the protein evaluation system (AfB, 1995; 1997). The efficiency of utilization of apparently digestible IAA for protein retention was highest for His and Met and amounted to 33 (Ile), 34 (Phe and Thr), 38 (Lys and Val), 41 (Leu), 52 (Met) und 76 (His) \%, respectively. The intersection of the regression curve (AA-deposition against apparently digestible AA) with the abscissae resulted in the maintenance requirement of absorbable amino acid. For growing bulls (BW: $300 \mathrm{~kg}$ ) a value of $4,7 \mathrm{~g} / \mathrm{d}$ histidine and 4,2 $\mathrm{g} / \mathrm{d}$ methionine was determined, but more investigations are necessary in this field.
\end{abstract}

Key Words: bulls, growing, amino acids, intestinum, utilization, maintenance requirement

\section{Zusammenfassung}

Die Bestimmung der Verwertung der im Intestinum scheinbar absorbierten Aminosăuren (AA) gestaltet sich schwierig, da nicht ausgeschlossen werden kann, dass das Angebot an einzelnen essentiellen Aminosaluren den Erhaltungs- und/oder Leistungsbedarf beeinflusst. Die Abhăngigkeit zwischen dem Aminosăurenansatz und dem Fluss essentieller absorbierbarer Aminosăuren ins Duodenum wurde an vier wachsenden Bullen (LM: 240-310 $\mathrm{kg}$ ) untersucht, um die Verwertungsraten dieser Aminosăuren zu kalkulieren. Die Tiere waren mit einer duodenalen T-Kanule versehen. Es wurden Rationen mit unterschiedlichen RP-Gehalten jedoch gleichbleibendem Energieniveau (11,2 MJ/kg DM) verfuttert. Die Verwertung der im Intestinum scheinbar absorbierten essentiellen Aminosäuren verringerte sich mit zunehmendem Ernăhrungsniveau. Dieses steht im Gegensatz zum gegenwärtig konstanten Verwertungsquotient des AfB $(1995 ; 1997)$. Beim zweifachen Erhaltungsbedarf betrug die Verwertung für Erhaltung und Ansatz 33 (Ile), 34 (Phe und Thr), 38 (Lys und Val), 41 (Leu), 52 (Met) und 76 (His) \%. Anhand der regressiven Auswertung der Abhăngigkeit zwischen dem AA-Ansatz und der Menge an scheinbar absorbierten essentiellen AA im Intestinum lassen sich Aussagen zum Erhaltungsbedarf dieser Aminosăuren treffen. Fur einen Bullen (LM: $300 \mathrm{~kg}$ ) wurde ein Erhaltungsbedarf von 4,7 g Histidin sowie 4,2 g Methionin je Tag errechnet.

Schlusselwörter: Bullen, Wachstum, Aminosăuren, Intestinum, Verwertung, Erhaltungsbedarf 
1. Einleitung

Die Effizienz der tierischen Leistung wird maßgeblich durch die Höhe der Verwertung der im Darm absorbierten Aminosäuren (AA) für Erhaltung und Leistung bestimmt. Eine exakte Quantifizierung dieser Größe ist aufgrund ungenügender Kenntnisse der komplexen Verwertungsprozesse wie der Beziehung zwischen Bedarf und Angebot einzelner AA oder der Wirkung limitierender AA für die Erhaltung bzw. das entsprechende Leistungsprodukt (Milch, Fleisch, Wolle, Konzeption) sowie der Wechselwirkung zur Energieversorgung schwierig. Diese Schwierigkeiten erklären die große Schwankungsbreite dieser Größe von 50 bis $80 \%$ in verschiedenen Proteinbewertungssystemen für Wiederkäuer (ALDERMAN, 1987).

Die Mehrzahl der Autoren, die unterschiedlichste experimentelle Methoden mit dem Ziel der Bestimmung dieses Parameters anwandten, gehen vorwiegend auf Untersuchungen mit Schafen und Kühen zurück (STORM und ØRSKOV, 1982; SMITH, 1984; WHITELAW et al., 1986; OLDHAM, 1987; ØRSKOV und CHEN, 1989). Eine Literaturzusammenstellung von ROHR und LEBZIN (1991) ergab eine Verwertung des AA-N für die Milchproduktion von 75 bis $80 \%$. Für wachsende Bullen wurde ein Wert von $67 \%$ abgeleitet. GABEL und POPPE (1988) ermittelten an ruminierenden Jungbullen im Lebendmasse- (LM) Abschnitt von 150 bis $200 \mathrm{~kg}$ eine abnehmende Verwertung der essentiellen Aminosäuren mit zunehmendem Ernährungsniveau von $76 \%$ beim Erhaltungsbedarf (EB) 1 auf $48 \%$ beim EB 4 . Beim präruminanten Kalb zwischen 80 und $240 \mathrm{~kg}$ LM fanden GERRITS et al. (1998) nur eine Verwertung der ileal verdaulichen essentiellen AA von unter $30 \%$.

Das Ziel der vorliegenden Untersuchungen bestand darin, die Verwertung der im Darm scheinbar absorbierten essentiellen AA bei wachsenden Bullen, die isoenergetisch mit variierenden Proteinmengen versorgt wurden, zu berechnen.

2.

Material und Methoden

Die Versuche wurden an vier Jungbullen der Rasse Schwarzbuntes Milchrind, die am Duodenum mit einer T-Kanüle aus Silikon $(25 \mathrm{~mm} \varnothing$, ca. $10 \mathrm{~cm}$ distal zum $D$. pancreaticus und $D$. choledochus) ausgerüstet waren, in einem LM-Abschnitt von 240310. kg durchgeführt. Die Tiere befanden sich auf Stoffwechselständen, die die quantitative Messung von Futteraufnahme sowie der Kot- und Harnausscheidungen ermöglichten. Die Umgebungstemperatur betrug 19 bis $22^{\circ} \mathrm{C}$. Die Fütterung der Tiere erfolgte zweimal täglich um 7.00 Uhr morgens und 16.00 Uhr nachmittags. Die Energiekonzentration der Ration betrug im Mittel 11,2 $\pm 0,4$ MJ Umsetzbare Energie (ME)/kg DM. Die Zusammensetzung der im Rohprotein(CP)-Gehalt variierenden Rationen ist in der Tabelle 1 aufgefürt.

Jede Versuchsperiode umfasste eine Vorperiode von $16 \mathrm{~d}$ als Adaptationsphase an das entsprechende RP-Fütterungsniveau, eine 7-tägige N-Bilanzmessung sowie eine anschließende Digestasammlung von $4 \mathrm{~d}$, in der Proben zur Bestimmung der Digestaflussraten am Duodenum genommen wurden. Das als Marker genutzte $\mathrm{TiO}_{2}$ wurde in pelletierter Form (5\% im Gerstenschrot) zweimal täglich vor Beginn der Fütterung zu je $5 \mathrm{~g}$ verabreicht. 
Die N-Bilanzmessung wurde auf der Grundlage der Richtlinien zur Verdaulichkeitsmessung (AfB, 1991) durchgeführt. Dabei wurde der Harn in Vorlagen gesammelt, deren pH-Wert durch den Zusatz von $6 \mathrm{~N}$ HCL unter 2.5 lag. Von der homogenisierten Tagesprobe wurden $5 \%$ für die Sammelprobe gepoolt, die der N-Bestimmung diente. Zur Messung des Digestaflusses wurden an 4 Tagen im Abstand von $4 \mathrm{~h} \mathrm{ca} .1000 \mathrm{~g}$ Digesta derartig entnommen, dass insgesamt zu jeder vollen Stunde eine Probe vorlag. Nach der Homogenisation der Probe wurden jeweils $100 \mathrm{~g} \mathrm{zu}$ einer Sammelprobe gepoolt und bei $0-4^{\circ} \mathrm{C}$ aufbewahrt. Der Rest der Probe wurde auf $37-39^{\circ} \mathrm{C}$ erwärmt und in das Duodenum zurückgeführt Am Ende der Sammelperiode wurde die gepoolte Probe homogenisiert und ein Teil wurde für die Bestimmung von $\mathrm{N}, \mathrm{NH}_{3}$ und der DM verwendet. $1500 \mathrm{~g}$ wurden lyophilized und durch ein 1-mm-Sieb gemahlen.

Die N-Bestimmung erfolgte nach der Kjeldahl-Methode (VON LENGERKEN und ZIMMERMANN, 1991). $\mathrm{TiO}_{2}$ wurde in Kot und Digesta im Kjeldahlaufschluß (Aufschlußdauer von $3 \mathrm{~h}$ ) photometrisch (Spekol 221, ZEISS, Jena) bei $405 \mathrm{~nm}$ bestimmt.

Tabelle 1

Zusammensetzung der Futterration ( $\mathrm{g} / \mathrm{kg} \mathrm{DM}$ ) (Composition of experimental diets, $\mathrm{g} / \mathrm{kg} \mathrm{DM}$ )

\begin{tabular}{lrrrr}
\hline Ration & 1 & 2 & 3 & 4 \\
\hline Winterweizen & 169 & 289 & 200 & - \\
Wintergerste & 164 & 118 & 158 & 83 \\
Tapiokamehl & - & - & 173 & - \\
Sojaextraktionsschrot & 172 & 89 & 206 & - \\
Trockenschnitzel & 38 & 147 & 23 & - \\
Trockengrunpellets & 58 & 193 & 40 & - \\
Wiesenheu & 196 & 109 & 188 & 216 \\
Gerstestroh & - & - & 12 & - \\
Weizenstroh & 195 & 9 & & 12 \\
Mineralstoffgemisch & 8 & 11,5 & 11,1 & 10,7 \\
& 11,1 & 156 & 102 & 63 \\
Energie (MJ ME/kg DM) & 200 & 172 & 156 & 158 \\
Rohprotein (g/kg DM) & 181 & & & - \\
Rohfaser (g/kg DM) & & & & \\
\hline
\end{tabular}

Als Standards dienten schwefelsaure Lösungen mit 20 und $60 \mathrm{mg} \mathrm{TiO}_{2}$ je Liter (BRANDT und ALLAM, 1987).

Die Bestimmung der Aminosäuren erfolgte nach dreistündiger saurer Hydrolyse bei $19,6 \cdot 10^{4} \mathrm{~Pa}$ und $134^{\circ} \mathrm{C}$ säulenchromatographisch mittels HPLC (Kationentrennsäule LCA-K06 (Grom Analytik + Analytic GmbH; Herrenberg-Kayh), 9 min bei $57^{\circ} \mathrm{C}, 7$ $\min$ bei $60^{\circ} \mathrm{C}, 32 \mathrm{~min}$ bei $80^{\circ} \mathrm{C}$; Flussrate $0,4 \mathrm{ml} / \mathrm{min}$ ). Die für die schwefelhaltigen Aminosäuren erforderliche Oxidation erfolgte durch ein Gemisch von $\mathrm{H}_{2} \mathrm{O}_{2}$ und Ameisensäure im Verhältnis 9:1.

Die Versuchsergebnisse wurden aufgrund der sich ändernden Lebendmassen der Tiere zum Zweck der Vergleichbarkeit auf die metabolische Lebendmasse bezogen und durch die Anpassung an die Funktion $y=a+b \cdot e^{x}(y: A A-A n s a t z ; x$ : im Intestinum scheinbar verdaute (sv) AA) verallgemeinert. Aus dem Schnittpunkt des AA-Ansatzes mit der Abszisse ergibt sich der AA-Erhaltungsbedarf der entsprechenden essentiellen AA. Anhand des gemessenes N-Ansatzes, der im Intestinum svAA (Passage am Duo- 
denum minus Exkretion im Kot) sowie unter Nutzung der AA-Zusammensetzung des Körperproteinzuwachses nach GABEL et al. (1988) wurde die Verwertung der einzelnen AA wie folgt berechnet:

Verwertung $1(\%)=\frac{\mathrm{AA}-\text { Ansatz }}{\text { sv AA im Intestinum }} \times 100$

Verwertung $2(\%)=\frac{\text { AA - Ansatz }}{\text { sv AA im Intestinum - sv AA im Intestinum für Erhaltung }} \times 100$

Differenzen zwischen den Mittelwerten wurden mittels einfaktorieller Varianzanalyse auf Signifikanz geprüft. Dafür und für die Berechnung der nichtlinearen Regression (Modell I) wurde das Programm SPSS (KÄHLER, 1992) herangezogen.

3.

Ergebnisse

Der N- und AA-Ansatz $\left(\mathrm{g} \cdot \mathrm{kg} \mathrm{LM}{ }^{-0,75} \cdot \mathrm{d}^{-1}\right)$ sowie die im Intestinum scheinbar verdauten essentiellen AA ( $\left.\mathrm{mg} \cdot \mathrm{kg} \mathrm{LM}^{-0,75} \cdot \mathrm{d}^{-1}\right)$ sind in den Tabellen 2 und 3 angegeben. Der N-Ansatz unterschied sich signifikant zwischen allen Rationen und lag in einem Bereich von $0,65 \mathrm{~g} \cdot \mathrm{kg} \mathrm{LM}^{-0,75} \cdot \mathrm{d}^{-1}(156 \mathrm{~g} \mathrm{CP} / \mathrm{kg} \mathrm{DM})$ bis $0,10 \mathrm{~g} \cdot \mathrm{kg} \mathrm{LM}^{-0,75} \cdot \mathrm{d}^{-1}(63 \mathrm{~g}$ $\mathrm{CP} / \mathrm{kg}$ DM). Der geringere N-Ansatz bei Ration 1 mit nur $0,51 \mathrm{~g} \cdot \mathrm{kg} \mathrm{LM}^{-0,75} \cdot \mathrm{d}^{-1}$ war im Vergleich zum N-Ansatz bei Ration 2 mit $0,65 \mathrm{~g} \cdot \mathrm{kg} \mathrm{LM}^{-0,75} \cdot \mathrm{d}^{-1}$ auf die höhere LM der Tiere während dieser Messperiode zurückzuführen. Der mit dem N-Ansatz in enger Beziehung stehende AA-Ansatz ergab für alle acht essentiellen AA bei Ration 2 die signifikant höchsten und bei Ration 4 die signifikant niedrigsten Werte, wobei Leu und Lys mit 279 bzw. $261 \mathrm{mg} / \mathrm{kg} \mathrm{LM}^{0,75}$ am meisten angesetzt wurden. Im Gegensatz dazu wurde Met über alle Rationen in einem Bereich von 88 (Ration 2) bis $13 \mathrm{mg} \cdot \mathrm{kg}$ $\mathrm{LM}^{-0,75} \cdot \mathrm{d}^{-1}$ (Ration 4) am geringsten angesetzt. Die Menge der im Intestinum scheinbar verdauten Aminosäuren nahm ab Ration 2 (156 g CP/kg DM) tendenziell ab. In der Periode der Niedrig-Protein-Ration (Ration 4, $63 \mathrm{~g} \mathrm{CP} / \mathrm{kg} \mathrm{DM}$ ) wurden bei allen Aminosäuren die geringsten AA-Mengen scheinbar verdaut.

Tabelle 2

$\mathrm{N}$ - und Aminosăurenansatz $(y \pm s ; n=4)$ (Retention of $N$ and $A A ;$ mean $\pm S D ; n=4)$

\begin{tabular}{lcccc}
\hline Ration & 1 & 2 & 3 & 4 \\
\hline CP $(\mathrm{g} / \mathrm{kg} \mathrm{DM})$ & 200 & 156 & 102 & 63 \\
DM-Aufnahme $(\mathrm{kg} / 100 \mathrm{kgLM})$ & $1,90^{\mathrm{a}} \pm 0,05$ & $2,29^{\mathrm{b}} \pm 0,10$ & $2,04^{\mathrm{b}} \pm 0,15$ & $1,83^{\mathrm{b}} \pm 0,11$ \\
N-Ansatz $\left(\mathrm{g} \cdot \mathrm{kg} \mathrm{LM}^{-0.75^{-1}} \cdot \mathrm{d}^{-1}\right)$ & $0,51^{\mathrm{a}} \pm 0,06$ & $0,65^{\mathrm{b}} \pm 0,05$ & $0,40^{\mathrm{b}} \pm 0,02$ & $0,10^{\mathrm{a}} \pm 0,03$ \\
AA & & $\mathrm{AA}-$ Ansatz $\left(\mathrm{g} \cdot \mathrm{kg} \mathrm{LM}^{-0.75} \cdot \mathrm{d}^{-1}\right)$ & \\
His & $111^{\mathrm{a}} \pm 13$ & $143^{\mathrm{ab}} \pm 12$ & $87^{\mathrm{b}} \pm 4$ & $22^{\mathrm{ab}} \pm 6$ \\
Lys & $202^{\mathrm{a}} \pm 24$ & $261^{\mathrm{ab}} \pm 22$ & $159^{\mathrm{b}} \pm 8$ & $40^{\mathrm{ab}} \pm 12$ \\
Leu & $216^{\mathrm{a}} \pm 25$ & $279^{\mathrm{ab}} \pm 23$ & $170^{\mathrm{b}} \pm 9$ & $43^{\mathrm{ab}} \pm 12$ \\
Phe & $111^{\mathrm{a}} \pm 13$ & $143^{\mathrm{ab}} \pm 12$ & $87^{\mathrm{b}} \pm 4$ & $22^{\mathrm{ab}} \pm 6$ \\
Met & $69^{\mathrm{a}} \pm 8$ & $88^{\mathrm{ab}} \pm 7$ & $54^{\mathrm{b}} \pm 3$ & $13^{\mathrm{ab}} \pm 4$ \\
Thr & $125^{\mathrm{a}} \pm 15$ & $162^{\mathrm{ab}} \pm 13$ & $98^{\mathrm{b}} \pm 5$ & $25^{\mathrm{ab}} \pm 7$ \\
Val & $145^{\mathrm{a}} \pm 17$ & $187^{\mathrm{ab}} \pm 16$ & $114^{\mathrm{b}} \pm 6$ & $29^{\mathrm{ab}} \pm 8$ \\
Ile & $99^{\mathrm{a}} \pm 12$ & $128^{\mathrm{ab}} \pm 11$ & $78^{\mathrm{b}} \pm 4$ & $19^{\mathrm{ab}} \pm 6$ \\
\hline
\end{tabular}

a,b,c: gleiche Buchstaben kennzeichnen signifikante Differenzen zwischen den Behandlungen $(p<0,05)$ 
Tabelle 3

Im Intestinum scheinbar verdauliche AA $\left(\mathrm{g} \cdot \mathrm{kg} \mathrm{LM}^{-0,75} \cdot \mathrm{d}^{-1} ; \mathrm{y} \pm \mathrm{s} ; \mathrm{n}=4\right.$ ) (Apparent intestinal digestible AA; $\mathrm{g} \cdot \mathrm{kg} \mathrm{BW} \mathrm{B}^{-0.75} \cdot \mathrm{d}^{-1} ;$ Mean $\pm \mathrm{SD} ; \mathrm{n}=4$ )

\begin{tabular}{lcccc}
\hline AA/Ration & 1 & 2 & 3 & \multicolumn{1}{c}{4} \\
\hline His & $180^{\mathrm{ac}} \pm 21$ & $183^{\mathrm{bd}} \pm 25$ & $115^{\mathrm{ab}} \pm 15$ & $79^{\mathrm{cd}} \pm 12$ \\
Lys & $164^{\mathrm{a}} \pm 65$ & $646 \pm 84$ & $430^{\mathrm{ab}} \pm 40$ & $301^{\mathrm{ab}} \pm 23$ \\
Leu & $667^{\mathrm{ac}} \pm 103$ & $632^{\mathrm{bd}} \pm 73$ & $425^{\mathrm{ab}} \pm 47$ & $300^{\mathrm{cd}} \pm 25$ \\
Phe & $418^{\mathrm{a}} \pm 60$ & $341 \pm 41$ & $302^{\mathrm{a}} \pm 33$ & $160^{\mathrm{ab}} \pm 29$ \\
Met & $188^{\mathrm{ac}} \pm 29$ & $158^{\mathrm{bd}} \pm 20$ & $105^{\mathrm{ab}} \pm 19$ & $72^{\mathrm{cd}} \pm 12$ \\
Thr & $449^{\mathrm{ac}} \pm 44$ & $461 \pm 63$ & $277^{\mathrm{ab}} \pm 36$ & $215^{\text {cd }} \pm 28$ \\
Val & $456^{\mathrm{ac}} \pm 53$ & $479 \pm 52$ & $294^{\mathrm{ab}} \pm 50$ & $222^{\text {cd }} \pm 31$ \\
lle & $407^{\mathrm{ac}} \pm 65$ & $382 \pm 59$ & $222^{\mathrm{ab}} \pm 32$ & $180^{\text {cd }} \pm 18$ \\
\hline a,b,c,d, : gleiche kleine Buchstabenkennzeichnen signifikante Differenzen $(\mathrm{p}<0,05)$ & &
\end{tabular}

4.

\section{Diskussion}

Die Abhängigkeit des AA-Ansatzes und ihrer Verwertung (1 und 2) von der entsprechenden AA-Menge, die im Intestinum scheinbar verdaut wurde, ist für das Lys in der Abbildung beispielgebend dargestellt. Der Schnittpunkt des Lysinsansatzes mit der Abzisse kennzeichnet den Erhaltungsbedarf an im Darm scheinbar verdaulichem Lysin. Er beträgt $266 \mathrm{mg} \cdot \mathrm{kg} \mathrm{LM}{ }^{-0,75} \cdot \mathrm{d}^{-1}$ Die Verwertung 1 steigt mit zunehmendem Lysinansatz zunächst an, erreicht bei einem Lysinansatz von $205 \mathrm{mg} \cdot \mathrm{kg} \mathrm{LM}^{-0,75} \cdot \mathrm{d}^{-1}$ und bei einer Menge von Lysin $532 \mathrm{mg}$ sv Lys $\mathrm{kg} \mathrm{LM}^{-0,75} \cdot \mathrm{d}^{-1}$ einen Wert von $38 \%$ und fällt danach wieder ab. Die Verwertung 2 erreicht beim Niveau des Erhaltungsbedarfes Werte von über $100 \%$ und nimmt mit zunehmendem Lysinansatz degressiv ab. Für die acht zu betrachtenden Aminosäuren sind in der Tabelle 4 alle Angaben zum Erhaltungsbedarf, Ansatz sowie für die Verwertung 1 und 2 zusammengestellt. Für praktische Belange ist der AA-Ansatz und die AA-Verwertung bei einem Vielfachen des AA-Erhaltungsbedarfes (EB) von 1,5 bis 2,5 von Bedeutung.

Die Verwertung 1 steigt mit dem AA-Ansatz der jeweiligen AA und erreicht bei einem Ernährungsniveau EB $\cdot 2$ das Maximum. Dieses trifft für alle acht essentiellen AA zu. Nach dem Erreichen dieses Maximums fällt die Verwertung $1 \mathrm{ab}$. Die höchste Verwertung wiesen His und Met mit 76 bzw. $52 \%$ auf. Auffällig ist, dass das His bzw. Met im Gegensatz zu den übrigen AA durch einen wesentlich stärkeren Anstieg der Verwertung 1 im Vergleich zum entsprechenden His- bzw. Met-Ansatz gekennzeichnet ist. Für die Verwertung 2 ergibt sich bei EB - 1 bei allen AA ein Wert von über 100 $\%$, was die Aussagekraft dieser Werte einschränkt, weil offensichtlich Wechselwirkungen im Stoffwechsel dieser AA auf der Ebene des Erhaltungsbedarfes und des Ansatzes bestehen, die mit der angewandten Methode nicht erfasst werden können.

Für His bzw. Met ergibt sich die höchste Verwertung beim EB · 1,5 mit 195 bzw. 137 $\%$. Mit zunehmendem Ernährungsniveau nimmt die Verwertung 2 ab und erreicht erst bei EB $\cdot 1,5$ bei Phe, Thr, Val und Ile eine Verwertung von unter $100 \%$. Ausnahmen bilden das His und Met, die trotz einer weiteren Abnahme der Verwertung 2 bei einem Ernährungsniveau von EB $\cdot 2$ im Gegensatz zu den übrigen AA weiterhin eine Verwertung von über $100 \%$ aufweisen.

$\mathrm{Zu}$ ähnlich hohen Verwertungsraten von über $100 \%$ beim His und Met gelangten SCHOOF et al. (2000) bei Untersuchungen an wachsenden Jungbullen in einem Le- 
Tabelle 4

Verwertung der im Intestinum scheinbar verdauten essentiellen AA (Efficiency of deposition of intestinal apparently digested IAA)

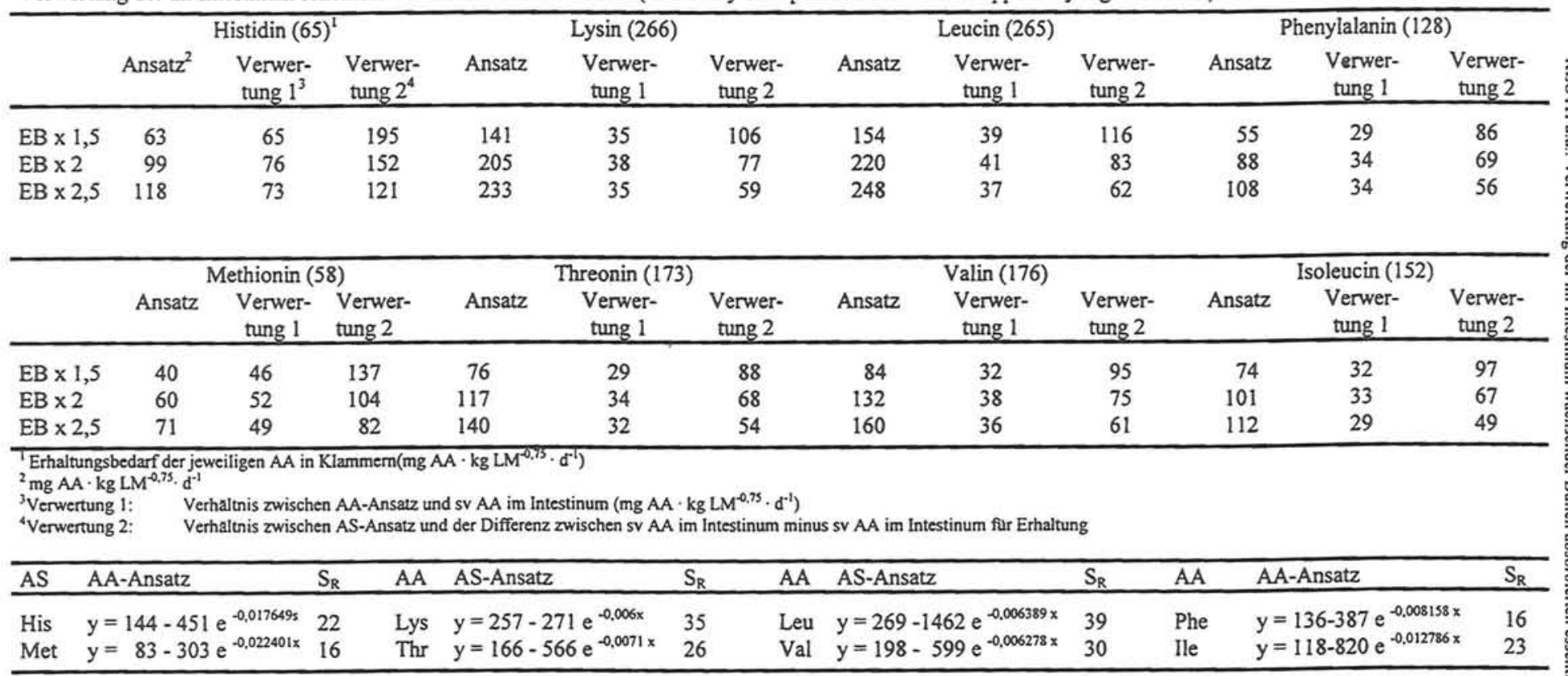


bendmasseabschnitt von 150 bis $200 \mathrm{~kg}$. Aus Untersuchungen von GABEL und POPPE (1988), die die Verwertung der einzelnen essentiellen scheinbar verdaulichen AA in Abhängigkeit vom Ernährungsniveau (Vielfache des energetischen Erhaltungsbedarfes) ermittelten, ergab sich gleichfalls eine abnehmende Verwertung dieser AA mit zunehmendem Ernährungsniveau, wobei His gefolgt von Lys und Leu die höchsten Verwertungsraten aufwiesen.

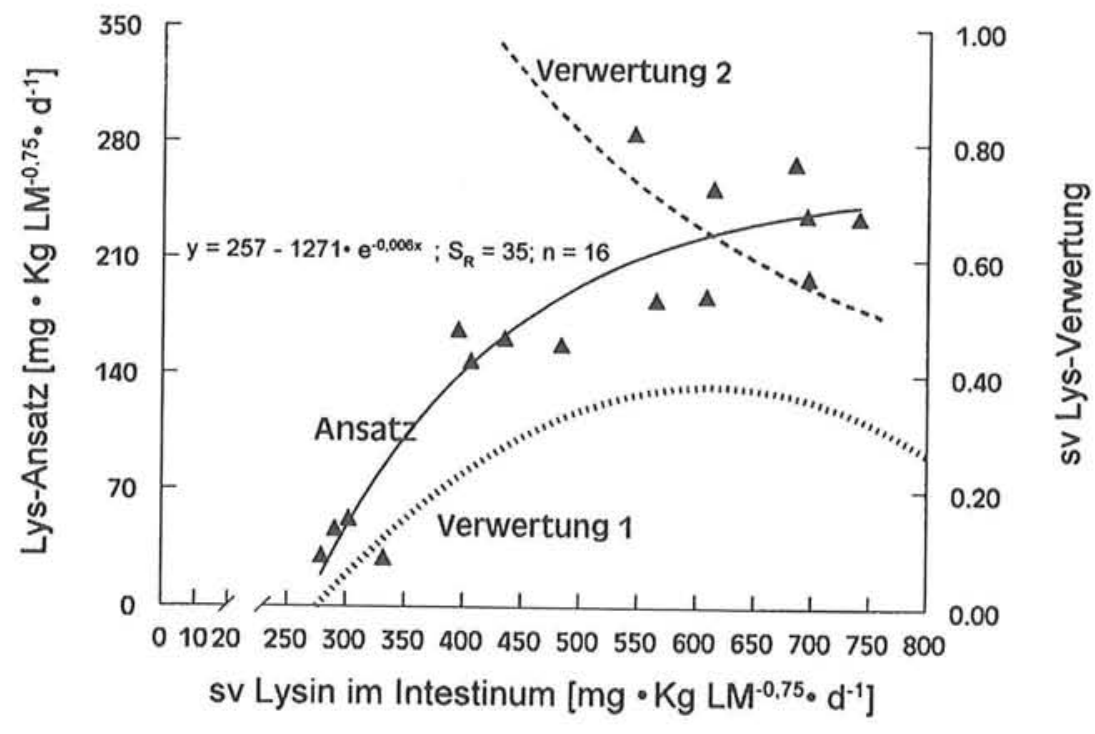

Abb.: Abhăngigkeit des Lysinansatzes vom scheinbar verdauten Lysin im Intestinum (Relationship between lysine deposition and intestinal apparently digested lysine)

VERITÉ et al. (1987) konnten aus Versuchen mit unterschiedlichen Rassen eine veränderliche Verwertung des absorbierten AA-N feststellen, die für Holstein-FriesianBullen (LM: 150-600 kg) bzw. Charolais-Bullen (LM: 400-750 kg) eine abnehmende Verwertung mit zunehmender Lebendmasse von 68 auf $28 \%$ bzw. 64 auf $30 \%$ ergab. Eine Ursache für die abnehmende Verwertung scheint die zunehmende AA-Überversorgung in höheren LM-Abschnitten zu sein (AfB, 1995).

Aus der höchsten für His und Met abgeleiteten Verwertung für den Proteinansatz ist zu folgern, dass His (GABEL und POPPE, 1986; TITGEMEYER et al., 1988; BLACK et al., 1990; MERCHEN und TITGEMEYER, 1992) und Met (GIBB et al., 1992) für das Wachstum eine colimitierende Wirkung besitzen.

Von SCHOOF et al. (2000) konnte diese limitierende Wirkung des His durch die NBilanztechnik (duodenale Infusion) nicht nachgewiesen werden. Eine Beeinflussung des intermediären Proteinumsatzes durch die duodenale Histidininfusion konnte dagegen mit der ${ }^{15} \mathrm{~N}$-Tracertechnik deutlich sichtbar gemacht werden (VOIGT et al., 1997), 
was die These des Vorhandenseins und der Wirkung von physiologischen Puffern wahrscheinlich erscheinen läßt. Entsprechende Puffer wären Hämoglobin, Anserin ( $ß$ Alanyl-1-Methylhistidin), Balanin (ß-Alanyl-3-Methylhistidin) und Carnosin ( $\beta$ Alanyl-Histidin), die während Phasen einer Histidinunterversorgung durch Histidinabgabe kompensierend auf die Mangelsituation im Stoffwechsel des Tieres wirken.

Die ermittelte abnehmende Verwertung der scheinbar verdaulichen essentiellen AA mit der Zunahme des Ernährungsniveaus steht im Gegensatz zur gegenwärtig vom AfB $(1995,1997)$ praktizierten Proteinbedarfskalkulation.

Durch die Beziehung zwischen dem AA-Ansatz und der jeweiligen im Intestinum scheinbar verdaulichen AA-Menge lassen sich unter dem Vorbehalt, dass andere spezifische Wirkungen der AA im Stoffwechsel des Tieres nicht beachtet werden, Aussagen zum Erhaltungsbedarf der einzelnen essentiellen AA treffen.

$\mathrm{Da}$ aus der Literatur nur wenige Angaben zum Erhaltungsbedarf verfügbar sind sollen die eigenen und die Ergebnisse von GABEL und POPPE (1988) sowie die Angaben von OWENS (1987), kalkuliert wurden, auf der Basis der metabolischen Lebendmasse verglichen werden (Tab. 5).

\section{Tabelle 5}

Erhaltungsbedarf an im Intestinum scheinbar verdaulicher essentieller AA $\left(\mathrm{mg} \cdot \mathrm{LM}^{-0.75} \cdot \mathrm{d}^{-1}\right)($ Requirement of intestinal apparently digestible IAA; $\mathrm{mg} \cdot \mathrm{BW}^{\cdot 0.75} \cdot \mathrm{d}^{-1}$ )

\begin{tabular}{lcccc}
\hline AS & OWENS $^{(1987)^{1}}$ & $\begin{array}{c}\text { GABEL \& POPPE } \\
(1988)\end{array}$ & eigene Untersuchungen ${ }^{2}$ & $\begin{array}{c}\text { Durchschnitt } \\
\text { Spalte } 3 \text { und } 4\end{array}$ \\
\hline His & 62 & 76 & 65 & 71 \\
Lys & 147 & 208 & 266 & 237 \\
Leu & 147 & 255 & 265 & 260 \\
Phe & 75 & 110 & 128 & 119 \\
Met & 75 & 76 & 58 & 67 \\
Thr & 75 & 155 & 173 & 164 \\
Val & 87 & 168 & 176 & 172 \\
Ile & 87 & 129 & 152 & 140 \\
\hline
\end{tabular}

'Berechnet nach CHALUPA (1974)

Der höchste Erhaltungsbedarf besteht laut Untersuchungen von GABEL und POPPE (1988) bei Leu mit $255 \mathrm{mg} / \mathrm{kg} \mathrm{LM}^{0,75}$ gefolgt von Lys mit $208 \mathrm{mg} / \mathrm{kg} \mathrm{LM}^{0,75}$, der niedrigste Erhaltungsbedarf bei His und Met mit jeweils $76 \mathrm{mg} \cdot \mathrm{LM}^{-0,75} \cdot \mathrm{d}^{-1}$. Aus den eigenen Untersuchungen ergeben sich gleichfalls für erstgenannte AA die höchsten Angaben, wobei Lys mit $266 \mathrm{mg} \cdot \mathrm{LM}^{-0,75} \cdot \mathrm{d}^{-1}$ nur geringfügig über dem Wert für Leu mit $265 \mathrm{mg} \cdot \mathrm{LM}^{-0,75} \cdot \mathrm{d}^{-1}$ liegt.

Während zwischen den Angaben von GABEL und POPPE (1988) und den eigenen Untersuchungen eine relativ gute Übereinstimmung besteht, werden bei einer Zusammenfassung beider Versuche erhebliche Unterschiede zu den Werten von OWENS (1987) offensichtlich.

Ausnahme bilden His und Met mit $62 \mathrm{bzw} .75 \mathrm{mg} \cdot \mathrm{kg} \mathrm{LM}{ }^{-0,75} \cdot \mathrm{d}^{-1}$, die gegenüber den zusammengefassten Ergebnissen mit $71 \mathrm{bzw} .67 \mathrm{mg} \cdot \mathrm{kg} \mathrm{LM}^{-0,75} \cdot \mathrm{d}^{-1}$ noch vergleichbar sind. Hingegen umfassen die Angaben von OWENS (1987) z. T. weniger als $50 \%$ der zusammengefassten Werte, wie es beim Thr deutlich wird. Die Ursachen für diese Dif- 
ferenzen dürften im unterschiedlichen methodischen Vorgehen zu suchen sein.

In den vorliegenden Untersuchungen wurden die im Dünn- und Dickdarm (Duodenum bis Rectum) scheinbar verdaulichen AA gemessen und für die Berechnung der AAVerwertung für den Körperproteinzuwachs zu Grunde gelegt. Infolge von unverdaulichem endogenen Protein am Ileum und Rectum (VAN BRUCHEM et al., 1997) ist die wahr absorbierte AA-Menge größer als die gemessene scheinbar verdauliche AAMenge. Diese Unterbestimmung der absorbierten AA fuhrt zu einer Überschätzung der AA-Verwertung. Dem wirkt jedoch der mikrobielle Abbau der am Ileum vorhandenen AA im Enddarm entgegen.

Für die als limitierend wirkend in Betracht kommenden AA His und Met wurden Angaben für den Erhaltungsbedarf von 65 und $58 \mathrm{mg} \cdot \mathrm{kg} \mathrm{LM}^{-0,75} \cdot \mathrm{d}^{-1}$ ermittelt, die absoluten Mengen bei einem $300 \mathrm{~kg}$ schweren Bullen von 4,7 $\mathrm{g}$ His und 4,2 g Met je Tag entsprechen.

Aus den Ergebnissen ist zu folgern, dass die Verwertung der essentiellen AA für Erhaltung plus Proteinansatz mit Ausnahme der von His und Met unter $40 \%$ liegt. Die Ursachen dieser geringen Verwertungshöhe sind weiter zu untersuchen. Die Verwertung für den Proteinansatz sinkt mit dem Ernährungsniveau. His und Met sind für das Wachstum als colimitierende AA anzusehen.

\section{Literatur}

AfB:

Ausschuß fur Bedarfsnormen der Gesellschaft für Ernăhrungsphysiologie. Leitlinien fur die Bestimmung der Verdaulichkeit von Rohnăhrstoffen an Wiederkăuern. J. Anim. Phys. a. Anim. Nutr. 65 (1991), 229-

AfB:

Ausschuß für Bedarfsnormen der Gesellschaft fur Ernăhrungsphysiologie. Energie- und Năhrstoffbedarf landwirtschaftlicher Nutztiere. Nr. 6, Empfehlungen zur Energie- und Năhrstoffversorgung der Mastrin-

AfB: der. DLG-Verlag, Frankfurt (Main) (1995)

\section{ALDERMAN, G.:}

Mitteilungen des Ausschusses fur Bedarfsnormen der Gesellschaft fur Ernăhrungsphysiologie. Zum Proteinbedarf von Milchkühen und Aufzuchtrindern. Proc. Soc. Nutr. Physiol. 6 (1997) 217-236

Comparison of rations calculated in different systems. In: JARRIGE, R. and G. ALDERMANN (Eds.): Feed evaluation and protein requirement systems for ruminants. Proc. CEC Seminar Brussels, 25 to 27 June 1986, ECSC-EEC-EAEC, Brussels (1987), 283-297

BLACK, A.; ANAUD, R. S.; BRUSS, M. L.; BROWN, A.; NAKAGIRI, J. A.:

BRANDT, M.; ALLAM, S.M.:

Partitioning of amino acids in lactating cows: oxidation to carbon dioxide. J. Nutr. 120 (1990), 700-710

Analytik von $\mathrm{TiO}_{2}$ im Darminhalt und Kot nach Kjeldahlaufschluß. Arch. Anim. Nutr. 37, 5 (1987), 453-454

CHALUPA; W.:

Amino acid nutrition of growing cattle. In: Tracer studies on non-protein-nitrogen for ruminants. Vienna 2 (1974), 175-194

GABEL, M.; POPPE, S.:

Intestinal amino acid supply in relation to the amino acid requirement in growing bulls. Arch. Anim. Nutr. 36; 2-3 (1986), 227-234 
GABEL; M.; POPPE, S.; PIEPER, B.:

Amino acid deposition in depence on energy level of the diet in fattening bulls (SMR). Wiss. Z. Uni. Rostock, N-Reihe 37, 2 (1988), 115

GABEL, M.; POPPE, S.: Untersuchungen uber die Verwertung der einzelnen essentiellen absorbierbaren Aminosäuren am Duodenum beim wachsenden männlichen Jungrind. Wiss. Z. Uni. Rostock, N-Reihe 37, 6 (1988), 3-7

GERRITS, W.J, SCHRAMA, J.W.; TAMMINGA, S.: The marginal efficiency of utilization of all ileal digestible indispensable amino acids for protein gain is lower than $30 \%$ in preruminant calves between 80 and $240 \mathrm{~kg}$ live weight. J. Nutr. 128 (1998) 1774-1785

GIBB, D.J.; KLOPFENSTEIN, T.J.; BRITTON; R.A.; LEWIS, A.J.:

KÄHLER, W. M.: Plasma amino acid response to graded levels of escape protein. J. Anim. Sci. 70 (1992), 2885-2892

LENGERKEN v., J.; ZIMMERMANN, K.; Handbuch Futtermittelprufung. Deutscher Landwirtschaftsverlag, Berlin; (1991), 172-176

MERCHEN, N. R.; TITGEMEYER, E.C.: Manipulation of amino acid supply to the growing ruminant. J. Anim. Sci. 70 (1992), 3238-3247

OLDHAM, J. D.: Efficiencies at amino acid utilization. In: JARRIGE, R. and G. ALDERMAN (Eds.): Feed evaluation and protein requirement for ruminants. Proc. CEC Seminar Brussel, 25.-27. june 1986 (1987), 171186

ØRSKOV, E. R.; B. CHEN, B.: Assessment of amino acid requirement in ruminants. Proc. Satellite Symp. of the $7^{\text {th }}$ Intern. Symp. Ruminant Physiology. Hakone, Japan (1989), 161-167

OWENS, F. N.: Maintenance protein requirements. In: JARRIGE, R. and G. ALDERMAN (Eds.): Feed evaluation and protein requirement for ruminants. Proc. CEC Seminar Brilssel, 25.-27. june 1986 (1987), 187-211

ROHR, K.; LEBZIN, P.: Present knowledge of amino acid requirement for maintenance and production. $6^{\text {th }}$ Intern. Symp. Protein Metabolism and Nutrition. Herning, Denmark (1991), 127-137

SCHOOF, Å; GABEL, M.; VOIGT, J.; SCHÖNHUSEN, U.; KLUTH, H.: Investigations on the influence of duodenal histidine infusion on nitrogen and amino acid turnover of growing German Holstein bulls. Arch. Anim. Nutr. 53 (2000) in press

SMITH, R. H.:

Essential amino acid requirements and rationing systems for ruminants. Proc. $6^{\text {th }}$ Intern. Symp. On Amino Acid. Polish Academy of Sci., Polish Scientific Publishers (1984), 319-329

STORM, E.;. ØRSKOV, E.R.:

Biological value and digestibility of rumen microbial protein in lamb small intestine. Proc. Nutr. Soc. 41 (1982), 78A

TITGEMEYER; E. C.; MERCHEN, N.R.; BERGER, L.L.; E. DEETZ. L.E.:

Estimation of lysine and methionine requirements of growing steers fed corn silage-based or cornbased diet. J. Dairy Sci. 71 (1988), 421-434

VAN BRUCHEM, J., VOIGT,J.; LAMMERS-WIENHOVEN,T.S.C.W.; SCHÖNHUSEN, U.; KETELAARS, J.J.M.H.; S. TAMMINGA, S: Secretion and reabsorption of endogenous protein along the small intestine of sheep: estimates derived from ${ }^{15} \mathrm{~N}$ dilution of plasma non-protein-N. Brit. J. Nutr. 77 (1997) 273-286

VERITÉ, R.; MICHALET-DOREAU, B.; CHAPOUTOT ; P. ;PEYRAND, J.L. ; POUCET, C. : Révision du système des protèines digestibles dans l'intestin (P.D.I.). Bull. Tech., C.R.Z.V. Theix, INRA, 70 (1987), 19-34

VOIGT, J.; SCHOOF, Ä; KRAWIELITZKI, K.; GABEL, M.; KLUTH, H.; HAGEMEISTER, H.: Stickstoff-Turnover beim wachsenden Rind - Einfluß einer duodenalen Histidin-Infusion. Proc. Soc. Nutr. Physiol. 6 (1997) 81

WHITHELAW, F.G.; MILNE, J.S.; ØRSKOV, E.R.; SMITH, J.S.: The nitrogen and energy metabolism of lactating cows given abomasal infusions of casein. Br. J. Nutr. 55 (1986), 537-556 
Akzeptiert: 11.10 .2000

\author{
Anschriften der Verfasser \\ Dr. HOLGER KLUTH \\ Martin-Luther-Universităt Halle/Wittenberg \\ Landwirtschaftliche Fakultăt \\ Institut fur Ernăhrungswissenschaften \\ Emil-Abderhaldenstraße 26 \\ D-06108 Halle/Saale
}

E-Mail: Kluth(@)landw.uni-halle.de

Prof. Dr. MARTIN GABEL

Universitat Rostock

Agrar- und Umweltwissenschliche Fakultat

Institut fur umweltgerechte Tierhaltung

Justus-von-Liebig-Weg 8

D-18059 Rostock

E-Mail: martin.gabel@agrarfak.uni-rostock.de

Dr. JÜRGEN VOIGT

Dr. ULRIKE SCHÖNHUSEN

Forschungsinstitut fur die Biologie landwirtschaftlicher Nutztiere

Wilhelm-Stahl-Allee 2

D-18196 Dummerstorf

E-Mail:voigt@fbn-dummerstorf.de

E-Mail:schoenhu@fbn-dummerstorf.de 
Buchbesprechung

Ultrasonography and Reproduction in Swine

Principles and practical applications

F. MARTINAT-BOTTE, G. RENAUD, F. MADEC, P. COSTIOU, M. TERQUI

104 Seiten, zahlreiche Abbildungen, INRA-Editions RD 10, 78026 Versailles cedex, France, 2000, ISBN 2-7380-0887-9, Preis $300 \mathrm{~F}$ (ca. 92,00 DM oder 46 Euro)

INRA-Editions@versailles.inra.fr

Durch die Verfugbarkeit kleinerer und stärkerer Ultraschallsonden in Verbindung mit kleinen tragbaren Ultraschallgeräten werden detaillierte Untersuchungen der verschiedenen Organsysteme auch beim Nutztier unter Praxisbedingungen möglich. Das Buch aus der Reproduktionsforschungsgruppe der INRA in Frankreich hat dazu einen hervorragenden Beitrag in Form dieses reich illustrierten Buches für das Fortpflanzungssystem beim weiblichen Schwein abgeliefert. Neben den Grundlagen der Ultraschalltechnik und einem kurzen Abriß der Anatomie und Physiologie der Reproduktion werden die Ultraschallbefunde wăhrend Brunstzyklus, Trăchtigkeit sowie der post partum und Laktationsphase im Vergleich mit Skizze und anatomischem Bild dargestellt. Weiterhin wird ausführlich auf Abnormalităten und die Anwendung im Herdenmanagement eingegangen. Auch die wichtigsten Artefakte von Ultraschallbildern werden im Detail besprochen und dargestellt, so dass insgesamt auch für den Anfänger eine solide Grundlage für einen Einstieg in diese Technologie gegeben wird. Sehr wertvoll sind die kurzen Zusammenfassungen und Schlußfolgerungen sowie die kurze, aber informative Liste mit weiterfuhrender Literatur am Ende eines jeden Kapitels fur ein vertiefendes Studium der jeweiligen Detailaspekte. Das aus dem Franz̋ssischen ins Englische übersetzte Buch ist gut lesbar und stellt sowohl für den Anfänger als auch für den Fortgeschrittenen in diesem zukunftsträchtigen Bereich einen wertvollen und deshalb sehr empfehlenswerten Beitrag dar, zumal die Kosten fur ein Buch in dieser hohen Qualitătsaufmachung akzeptabel sind. 